\title{
Obesidad materna y riesgo de parto prematuro (1)
}

\author{
Cnattingius S, Villamor E, Johansson S, Edstedt Bonamy AK, Persson M, Wikström AK, \\ Granath F. Maternal obesity and risk of preterm delivery. JAMA 2013 12;309(22):2362- \\ 70.
}

Análisis Crítico: Jorge Carvajal C. ${ }^{1}$, PhD. Claudio Vera P-G. ${ }^{1,2}$, MSc.

${ }^{1}$ Unidad de Medicina Materno-Fetal, División de Obstetricia y Ginecología; ${ }^{2}$ Unidad de Medicina Basada en Evidencia. Escuela de Medicina. Pontificia Universidad Católica de Chile

\section{RESUMEN (1)}

Importancia: El parto prematuro es la principal causa de mortalidad, morbilidad y discapacidad a largo plazo en el período infantil, y estos riesgos aumentan a medida que disminuye la edad gestacional. La obesidad aumenta el riesgo de parto prematuro, pero la asociación entre el sobrepeso y la obesidad y subtipos de parto prematuro no son claras. Objetivo: Estudiar la asociación entre el índice de masa corporal (IMC) al comienzo del embarazo temprano y el riesgo de parto prematuro por edad gestacional y por precursores de parto prematuro. Método: Estudio de cohorte de base poblacional de mujeres que tuvieron partos únicos con hijos vivos en Suecia entre 1992 y 2010. Las características maternas y del embarazo se obtuvieron del Registro Médico Nacional de Nacimientos de Suecia. Principales resultados y medidas: Riesgo de parto prematuro (extremadamente prematuro, 22-27 semanas; muy prematuro, 28-31 semanas, y moderadamente prematuro, 32-36 semanas). Estos resultados fueron adicionalmente caracterizados como parto prematuro espontáneo (relacionado con contracciones pretérmino o rotura prematura pretérmino de membranas) y parto prematuro por indicación médica (parto por cesárea antes del inicio del trabajo de parto o trabajo de parto inducido). Las estimaciones de riesgo se ajustaron por edad materna, paridad, tabaquismo, educación, estatura, país de nacimiento de la madre y año del parto. Resultados: De los 1.599.551 partos con información sobre el IMC al inicio del embarazo, 3.082 eran extremadamente prematuros, 6.893 eran muy prematuros y 67.059 eran moderadamente prematuros. Los riesgos de parto extremadamente, muy y moderadamente prematuro aumentaron con el IMC, y el riesgo de parto prematuro relacionado con sobrepeso y obesidad fue mayor para parto extremadamente prematuro. Entre las mujeres con peso normal (IMC de 18,5 $-<25$ ), la tasa de parto extremadamente prematuro fue del $0,17 \%$. En comparación con las mujeres de peso normal, las tasas (\%) y Odds Ratio ajustado (OR [IC95\%]) de parto extremadamente prematuro fueron los siguiente: IMC de 25 a menos de 30 $(0,21 \%$, OR 1,26; IC95\% 1,15-1,37), IMC de 30 a menos de 35 (0,27\%, OR 1,58; IC95\% 1,39-1,79), IMC de 35 a menos de 40 (0,35\%, OR 2,01; IC95 $\% 1,66-2,45)$, e IMC de 40 o más $(0,52 \%$, OR 2,99; IC95\% 2,28-3,92). El riesgo de parto espontáneo extremadamente prematuro aumentó con el IMC entre las mujeres obesas (IMC $\geq 30$ ). Los riesgos de los partos prematuros médicamente indicados aumentaron con el IMC entre las mujeres con sobrepeso y obesidad. Conclusiones: En Suecia, el sobrepeso y la obesidad materna durante el embarazo se asocian con un mayor riesgo de parto prematuro, especialmente partos extremadamente prematuros. Estas asociaciones deben ser evaluadas en otras poblaciones.

\section{ANÁLISIS DE LA INVESTIGACIÓN}

\section{A. Relevancia clínica de la investigación}

Si se excluyen las malformaciones congénitas, el $75 \%$ de las muertes perinatales y el $50 \%$ de las anormalidades neurológicas de la infancia son atribuibles directamente a la prematurez (2). La morbilidad neonatal de los prematuros es de magnitud 
variable, dependiendo principalmente del peso de nacimiento, de la edad gestacional al nacer, de la existencia de malformaciones fetales, de la etiología responsable del parto prematuro, y de las características de la unidad de cuidado intensivo que atienda al recién nacido. Afortunadamente la gran mayoría de los prematuros se encuentran por sobre las 34 semanas, un período en que la morbilidad neonatal es habitualmente escasa y leve. Aproximadamente un $5 \%$ de los partos prematuros ocurren antes de las 28 semanas (prematuros extremos), $15 \%$ entre $28-31$ semanas (prematuro severo), cerca del $20 \%$ entre 32 y 33 semanas (prematurez moderada) y $60-70 \%$ entre $34-37$ semanas (cercano al término) (2). Los esfuerzos deben estar dirigidos a prevenir la prematurez extrema y severa, ya que esos recién nacidos son los que concentran la mayor parte de las secuelas entre los sobrevivientes $(3,4)$.

Obesidad y sobrepeso constituyen un grave problema en la población chilena actual, incluyendo al grupo de mujeres en edad reproductiva. La Encuesta Nacional en Salud del 2010 muestra que en el grupo de mujeres en edad reproductiva (1545 años), un $67,6 \%$ presenta sobrepeso u obesidad (33,6\% IMC 25-29,9; 30,7\% IMC 30,0-39,9 y 3,3\% IMC >40,0 aproximadamente) (5). Según el DEIS, en la población de embarazadas en Chile, el 53\% presenta sobrepeso u obesidad (6).

Dada la importancia del parto prematuro, es necesario buscar medidas eficientes de prevención, especialmente evitar los factores de riesgo prevenibles. Entre los factores de riesgo prevenibles para parto prematuro, se ha propuesto la existencia de una fuerte asociación entre sobrepeso/obesidad materna y el riesgo de parto prematuro. Se comprende la importancia de cuantificar y verificar la existencia de esta asociación de riesgo.

\section{B. El estudio (1)}

Diseño: Estudio observacional de cohorte de base poblacional efectuado en Suecia. Población incluida: Mujeres que tuvieron parto de hijo vivo, producto de un embarazo único, entre los años 1992-2010. Fuente de datos: Registro Médico Nacional de Nacimientos de Suecia, el que incluye todos los datos necesarios para el estudio, especialmente el peso y talla de la madre al inicio del control prenatal. El registro posee el dato de la edad gestacional, calculada por fecha de última menstruación o ecografía. Resultados medidos: Número de partos prematuros, categorizados en extremadamente prematuros (22-27 semanas), muy prematuro (28-31 semanas), moderadamente prematuro (32-36 semanas). Resultados obtenidos: En el período estudiado, la tasa global de parto prematuro (<36 semanas) fue $5,03 \% ; 0,23 \%$ (22-27 sem); 0,47\% (28-31 sem) y $4,36 \%$ (32-36 sem). Comparado con mujeres de peso normal, el riesgo de parto prematuro (en todos los rangos) aumenta a mayor IMC. La asociación fue especialmente alta para los partos extremadamente prematuros (22-27 semanas) (Tabla I).

\section{Análisis crítico}

Validez interna: En este tipo de diseño el riesgo de sesgo de información es difícil de dimensionar puesto que la información fue extraída de una base de datos; sin embargo, se explica que los datos son generados de modo prospectivo, y que una copia de ellos es enviado al registro central, haciendo del Registro Médico Nacional de Nacimientos de Suecia una fuente muy confiable de datos. La base de datos posee los datos de factores confundentes (tabaquismo, talla materna, nivel educacional), los que son considerados en el análisis para calcular

Tabla I

RIESGO DE PARTO PREMATURO 22-27 SEMANAS E IMC

\begin{tabular}{|c|c|c|c|}
\hline IMC & $\%$ parto $22-27$ sem & OR ajustado & IC95\% \\
\hline $18,5-<25,0$ & 0,17 & \multicolumn{2}{|c|}{ Referencia } \\
\hline $25,0-<30,0$ & 0,21 & 1,23 & $1,13-1,35$ \\
\hline $30,0-<35,0$ & 0,27 & 1,53 & $1,35-1,74$ \\
\hline $35,0-<40,0$ & 0,35 & 1,97 & $1,62-2,40$ \\
\hline$>40,0$ & 0,52 & 2,91 & $2,21-3,83$ \\
\hline
\end{tabular}


los OR ajustados. El número de datos perdidos o faltantes es escaso; para evitar este error, los autores efectúan un análisis de imputaciones múltiples, cuyos resultados son similares al análisis de los datos de los casos completos. No encontramos fallas en la validez interna del estudio. Validez externa: El estudio representa a la población de Suecia, $83 \%$ de las mujeres son de origen nórdico, por cierto, diferentes a las de nuestro medio. Se reporta un $29 \%$ de mujeres con sobrepeso/obesidad, lo que representa la mitad de nuestra tasa de sobrepeso/ obesidad. En resumen, es un estudio cuyos resultados son consistentes con reportes previos, en el cual se explora una asociación entre el factor de riesgo y el resultado final evaluado; se evidencia la asociación y se observa una relación dosis-respuesta. Conclusión: Se trata de un estudio observacional de cohorte de base poblacional, con muy bajo riesgo de sesgo, que muestra una asociación entre el IMC pre-embarazo de mujeres de Suecia y el riesgo de parto prematuro. Este estudio apoya la asociación entre obesidad pre-embarazo y el riesgo de parto prematuro, sugiriendo que el control de este factor prevenible podría reducir el riesgo de parto prematuro. Nos parece que es recomendable el control de peso materno como parte del consejo preconcepcional.

\section{REFERENCIAS}

1. Cnattingius $S$, Villamor $E$, Johansson $S$, Edstedt Bonamy AK, Persson M, Wikström AK, Granath F. Maternal obesity and risk of preterm delivery. JAMA 2013;12;309(22):2362-70.

2. Goldenberg RL, Culhane JF, lams JD, Romero R. Epidemiology and causes of pretermbirth. Lancet 2008;5;371(9606):75-84.

3. Saigal S, Doyle LW. An overview of mortality and sequelae of preterm birth from infancy to adulthood. Lancet 2008;371:261-9.

4. Tyson JE, Parikh NA, Langer J, Green C, Higgins RD. Intensive care for extreme prematurity-moving beyond gestational age. N Engl J Med 2008;358:1672-81.

5. Encuesta Nacional en Salud. MINSAL. Disponible en: http://web.minsal.cl/portal/url/item/bcb03d7bc28b64dfe040010165012d23.pdf. Acceso el 19 de enero 2014.

6. MINSAL. Disponible en: http://www.dipres.gob.cl/595/ articles-107673_doc_pdf.pdf. Acceso el 19 de enero del 2014. 